\title{
De la teoría y la aplicación lingüística a la construcción de la dignidad y los derechos humanos
}

\section{- From Linguistic Theory and ApplicationTowards the Construction of Dignity and Human Rights}

Ana María Rodino'

Resumen: La ciencia lingüística no dejó una marca particular, fácilmente identificable en el constructo de dignidad humana consagrado en la Declaración Universal de los Derechos Humanos. Su contribución fue más bien indirecta, inspiradora y principista, pero significativa porque hizo un doble aporte. Primero, se sumó a otras fuentes que respaldaron el discurso de los derechos humanos en la idea de dignidad como rasgo distintivo de los seres humanos. Segundo, contribuyó al razonamiento dialéctico que ese discurso construyó entre semejanza y diferencias para articular las dimensiones de la persona: miembro de la especie, integrante de una sociedad, e individuo autónomo. Sobre lo primero, la Lingüística y la Sociolingüística ofrecieron conocimientos científicos sobre las particularidades de la especie humana que hacen a sus miembros seres dignos de reconocimiento y protección. Enfatizaron nuestra unidad como especie singular (homo loquens, hombre que habla) a la vez que distinguieron nuestras diferencias como individuos y grupos sociales hablantes de distintas lenguas. Así, el estudio científico del lenguaje y las lenguas permitió conocer mejor a sus hablantes, sus facultades y creaciones, y a caracterizarlos como individuos libres, iguales y también cooperativos, en cuanto constructores de sociedades.

Sobre lo segundo, estas disciplinas ejemplificaron un discurso elaborado que reconoció nuestra semejanza esencial como familia humana -la facultad del

1 Licenciada y Profesora en Letras por la Universidad de Rosario, Argentina. Máster en Lingüística por la Universidad de Costa Rica, Costa Rica. Máster y Doctora en Educación por la Universidad de Harvard, Estados Unidos. anamariarodino@gmail.com 
lenguaje- en oposición y a la vez en complementación con nuestras diferencias evidentes como sociedades e individuos hablantes -las distintas lenguas naturales del planeta y las distintas variedades de cada lengua natural-. Articularon los dos reconocimientos en un discurso dialéctico e integrador, que no negó las tensiones entre ellos, pero tampoco las presentó como irresolubles. Las explicó y propuso políticas superadoras, sociales y educativas. De tal forma impulsaron su desarrollo como ciencias y también el discurso de los derechos humanos.

Palabras clave: Lingüística. Sociolingüística. Lingüística educativa. Dignidad. Derechos humanos. Pandemia.

Abstract: Linguistic science did not leave a particular, easily detectable mark in the human dignity construct acknowledged by the Universal Declaration of Human Rights. Its contribution was mainly indirect, inspirational, and of principle but significant since it made a double contribution. First, it added to other sources supporting the human rights discourse in the notion of dignity as a distinctive feature of human beings. Second, it contributed to the dialectical reasoning the human rights discourse developed between similarity and differences in order to articulate the various dimensions of a person: member of the human species, participant of a society, and autonomous individual. About the first, Linguistics and Sociolinguistics put forward scientific knowledge about the particularities of the human species that make its members creatures deserving recognition and protection. They emphasized our unity as a singular species (homo loquens, speaking man) while at the same time distinguished our differences as individuals and social groups speaking different languages. Therefore, the scientific study of language and natural languages allowed a better understanding of their speakers, their faculties and creations, and their portrayal as individuals who are free, equal and also cooperative as society builders.

About the second, these scientific disciplines displayed a complex discourse recognizing our essential similarity as members of the human family -the faculty of language - in contrast with our visible differences as societies and speakers -the different world languages, and the different varieties within each language. Both recognitions were articulated in an including and dialectic discourse, which neither denied the tensions between them nor presented them as unsolvable. Such a discourse explained the tensions while proposing comprehensive social and educational policies to deal with them. In such a way these sciences further their development, and the development of the 
human rights discourse.

Key words: Linguistics. Sociolinguistics. Educational Linguistics. Dignity. Human Rights. Pandemic

\section{El porqué de estas reflexiones... (continúa al final)}

El lenguaje fue mi amor primero, intenso y perdurable. No me abandonó nunca a lo largo de mi vida. En realidad, debería decir que yo nunca lo abandoné: lo estudié y practiqué apasionadamente y él me correspondió concediéndome una riqueza de relaciones, meditaciones, disfrutes, creaciones y provocaciones.

Claro, no solo a mí... El lenguaje nos abre a todos infinidad de posibilidades de pensamiento y acción. Porque está en todo lo que hacemos. Es el mediador de nuestras relaciones con nosotros mismos y con los demás en cada momento de la existencia: nos guía en la introspección, el juicio crítico, la producción artística, los vínculos afectivos, las labores del trabajo y la participación ciudadana -por citar sólo algunas de nuestras prácticas vitales. Está en todos los cruces posibles entre el mundo individual y el social.

Mi amor por el lenguaje me condujo a la Lingüística. Por eso este texto reúne apuntes y reflexiones desde mi perspectiva de lingüista buscando caminos que me acerquen a mi otro gran amor y compromiso profesional: la Educación en Derechos Humanos. Digo, apenas algunos caminos relevantes, porque es ilusorio querer abarcar la vastedad de ambas disciplinas en unas pocas páginas.

A continuación exploraré, primero, lo que considero aportes teóricos, aunque indirectos, de la ciencia lingüística y una de sus ramas a la noción de dignidad humana. Después, destacaré sus contribuciones interdisciplinarias directas al concepto más relevante que derivamos de la noción de dignidad humana: los derechos humanos.

\section{Las miradas teóricas de la Lingüística y la Sociolin- güística}

En occidente, el interés por el lenguaje es antiguo. Desde que nació dentro de la filosofía griega motivó muchos trabajos, por mucho tiempo de carácter filosófico-especulativo y después, ya en el siglo XIX, de carácter histórico y comparado, centrados en el origen y evolución de las formas lingüísticas, principalmente las europeas, y su grado de superioridad o perfección. Pero la preocupación realmente científica por el lenguaje y la lengua en sí mismos, como campo de estudio autónomo, llega en las primeras décadas 
del siglo XX con la corriente del estructuralismo lingüístico, que funda la Lingüística moderna. ${ }^{2}$ Esta corriente inicia el estudio sistemático de las lenguas en su realidad y sus estructuras actuales -no en su evolución ni comparándolas entre sí para jerarquizarlas. Es decir, su estudio formal como códigos.

La Lingüística moderna tiene un objeto de estudio doble: es ciencia del lenguaje -facultad característica, universal e inmutable de la especie homo sapiens-y de la lengua o las lenguas naturales -siempre particulares y variables, pues son productos de sociedades humanas concretas, resultantes de las interacciones acumuladas entre los individuos, independientemente de los genes. ${ }^{3}$ Esta disciplina le concede igual interés y valor a todas los lenguas, escritas o no, europeas o no, y desarrolla métodos rigurosos para describirlas. Y en su trabajo por comprender las lenguas, ayuda a comprender a sus hablantes: los seres humanos.

La facultad humana del lenguaje, materializada en las lenguas, media entre el individuo y la sociedad. Ninguna sociedad es posible sin la acción de la lengua y tampoco el sujeto es posible sin ella. Como ejemplifica un lingüista, el despertar de la conciencia en los niños y niñas coincide con el aprendizaje de su lengua materna, la cual los introduce poco a poco como individuos en su sociedad de origen. ${ }^{4}$

El lenguaje tiene tal rol fundacional porque constituye la forma más elevada de una capacidad propia de la condición humana: la de simbolizar. Simbolizar permite formar los conceptos (una representación) como algo diferente a los objetos de la realidad. Pensar es poder construir y operar con representaciones mentales. Esta capacidad que está en la base de las operaciones conceptuales no aparece más que en el ser humano: el salto de la función sensorial a la función simbólica sólo lo ha dado, hasta la fecha, nuestra especie.

La capacidad simbólica del lenguaje sirve al sujeto para variados usos o funciones: transmitir información sobre referentes de su contexto, del mundo real o del pensamiento (función referencial o informativa); expresar su subjetividad y sentimientos (función expresiva o emotiva); apelar a su/s oyente/s buscando influir en ellos (función apelativa); pensar sobre el propio lenguaje (función metalingüística); producir creaciones estéticas con el lenguaje (función poética o estética) y crear o mantener abierto el canal de comunicación con otro/s (función relacional o fática). ${ }^{5}$ Aún esta escueta revisión nos da una

2 Su comienzo se marca generalmente con la publicación del Curso de Lingüística General de Ferdinand de Saussure (1916), que da inicio a la corriente del estructuralismo lingüístico.

3 BENVENISTE, Emile [1966], Problemas de Lingüística General, Tomo I. Siglo XXI, México DF, 1971; MATTHEWS, H. P. Linguistics: A Very Short Introduction, New York: Oxford University Press. 2003.

4 BENVENISTE, Emile. Op. cit

5 JAKOVSON, Roman. Linguistics and Poetics. En: SEBEOK, Thomas A. Style In Language. Cambridge, Massachu- 
buena idea de las múltiples labores del lenguaje, facultad poderosa que habilita a los individuos a construir su identidad, comunicarse, pensar, aprender y cooperar con incontables otros individuos, aun los desconocidos, en pos de metas comunes, algunas tan abstractas como crear estados, religiones 0 sistemas de justicia. ${ }^{6}$ En este proceso se fundan las sociedades y las culturas. Pero si la facultad del lenguaje es una sola, común a todos los seres humanos, las lenguas naturales creadas por las sociedades humanas son muchas y muy diversas. Sin embargo, todas las lenguas (idiomas) del mundo comparten el ser códigos, es decir, sistemas de signos articulados, formados por unidades que se definen por las relaciones que guardan entre sí, cuyo número limitado permite construir significados ilimitados.

Las lenguas del mundo son todas flexibles por igual, aptas para cumplir las diferentes funciones del lenguaje, e interesantes y merecedoras de atención científica. La estructura de cada lengua puede ser más simple o más compleja según el tipo de signos que emplee y sus reglas para combinarlos, pero estos son rasgos descriptivos de su organización interna, no de valor. La expansión territorial de cada lengua puede haber originado que tengo un mayor o menor número de variantes lingüísticas, pero este es un dato de su historia, no un indicador de rango frente a otras lenguas. En la Lingüística no tienen cabida juicios de valor o jerárquicos entre lenguas, sus hablantes o sus culturas. Lo dice con claridad un notable lingüista y antropólogo: "no importa en qué nivel de la cultura se encuentran tipos de lenguas simples y complejos con número infinito de variedades, que vistas las cosas desde aquí no hay diferencia, por tratarse de la misma lengua, entre un criador de cerdos macedonio y Platón."7

La Lingüística se suma así a la Antropología para rebatir nociones previas fuertemente eurocentristas, que hoy día consideramos racistas. Una, la de "cultura" entendida como las formas superiores de las bellas artes y las humanidades -reservadas a sociedades "superiores" - para definirla como el conjunto de bienes materiales y espirituales de cada sociedad, incluyendo su lengua, saberes, creencias, pautas de conducta, tradiciones, valores y herramientas. Otra, la de "lenguas de cultura" versus "lenguas primitivas", estas últimas habladas por pueblos supuestamente "primitivos" o"salvajes", como con frecuencia se describía a aquellos que fueron sometidos por la conquista europea del mundo.

Comprender que el lenguaje, realizado en la lengua, hace la mediación entre individuo y sociedad condujo lógicamente al interés por estudiar de

setts: MIT Press. 1960.

6 HARARI, Yuval Noah. Sapiens. A Brief History of Mankind. New York: Harper Collins. 2015.

7 Cita de Edward Sapir reproducida en BENVENISTE, E. [1974], Problemas de Lingüística General, Tomo II. México DF: Siglo XXI, 3. ed., 1979. p. 96. 
manera científica esta relación: cómo funciona cada lengua en el contexto social concreto en que se aprende y se usa. La inquietud da origen, hacia mediados del siglo XX, a una rama de la Lingüística que estudia las interrelaciones entre lengua y sociedad. Se la conoce como Sociolingüística y cubre un amplio espectro de temas y problemas, analizados con diversas perspectivas, al punto que puede asumir otros nombres como Sociología del lenguaje o Etnolingüística.

La teoría sociolingüística se pregunta qué sucede con la lengua en los usos concretos de los hablantes mientras a sus necesidades comunicativas. Cuando lo hace, comprueba que el carácter social más notable de cualquier lengua (y de todas) es que no se manifiesta de una manera única y homogénea sino, al contrario, de maneras variadas. En otras palabras, presenta variantes de uso. De allí que una definición más exacta de la Sociolingüística es decir que estudia la diversidad y la variedad al interior de las lenguas.

Las lenguas varían de acuerdo con cuatro variables. Cambian a través del tiempo (variantes diacrónicas), del espacio (dialectos), de los grupos sociales (sociolectos) y de las situaciones sociales en las que se usan (registros). En breve, según uno de sus grandes representantes, la Sociolingüista se dedica a tratar de determinar quién habla cuál variedad de cuál lengua, cuándo, a propósito de qué y con cuáles interlocutores. ${ }^{8}$

Las variantes que describen los estudios sociolingüísticos no implican evaluación de unas frente a otras. No las hay mejores ni peores; son distintas pero todas equivalentes desde la mirada científica. Si existen juicios despectivos hacia determinadas variantes de una lengua en una sociedad - que sí existen - son los que crea la misma sociedad por razones no lingüísticas sino relativas a los hablantes que las usan (valoraciones según región geográfica, clase, nivel económico, raza o etnia, educación u ocupación, entre otros factores) o a las situaciones en que se usan (por ejemplo, en una conferencia académica versus un partido de fútbol).

Menospreciar alguna o algunas variantes de una lengua - o a lenguas minoritarias dentro de un Estado - se ha justificado siempre con argumentos valorativos: $X$ variante o lengua es más pobre, más rústica, menos comprensible, menos expresiva, menos elegante... Estos argumentos no tienen base científica, sus razones son extra-lingüísticas y hay que buscarlas en las diferencias y animosidades entre distintos grupos sociales dentro de cada Estado. En suma, no hay lengua homogénea: existen muchas variedades de lengua, cada una con sus reglas de uso. Desde esta óptica, es falso postular una norma "correcta" frente a otras incorrectas, erradas, descuidadas o antigramaticales. Y el juicio tan usado de "errores en el uso de la lengua" debe verse desde otra 
perspectiva. El sociolingüista entiende que no hay modos de hablar correctos o errados, gramaticales o antigramaticales, sino solamente modos de hablar "diversos", pertenecientes a variedades distintas de la lengua.

\section{Aportes lingüísticos y sociolingüísticos al concepto de dignidad humana}

Los argumentos disciplinarios que resumí no dejaron una marca directa específica, que se pueda identificar a primera vista en el concepto de dignidad humana consagrado en la Declaración Universal de los Derechos Humanos. Sostengo que su contribución fue más bien indirecta, inspiradora y principista, pero es significativa porque hizo un doble aporte. Por un lado, a las fuentes teóricas que respaldaron el discurso contemporáneo de los derechos humanos en la idea de dignidad como rasgo propio y distintivo de los seres humanos. Por otro, a la dialéctica que ese discurso construyó entre unidad y diversidad -o, en otras palabras, entre semejanza y diferencia- para articular las dimensiones de la persona: miembro de la especie, integrante de la sociedad e individuo autónomo. ${ }^{9}$

En cuanto al primer aporte, los enfoques y comprobaciones teóricas de la Lingüística y la Sociolingüistica, sumados a los de la Antropología, ofrecieron conocimientos científicos sólidos sobre las singularidades de la especie humana y de sus miembros, singularidades que la diferenciaban de otras especies animales y hacían a su condición de seres dignos de reconocimiento y protección. En breve, la ciencia lingüística aportó a que se comprendiera que:

- El lenguaje articulado, con su distintiva y excepcional capacidad simbólica, es una facultad exclusivamente humana.

- El lenguaje cumple variedad de funciones que responden a los requerimientos de todos los seres humanos, sin exclusiones ni diferenciaciones al interior de la familia humana;

- Las lenguas naturales son producto de la facultad del lenguaje, pero construidas por cada sociedad; por ello son tan diversas como lo son las mismas sociedades humanas del planeta.

- Las lenguas naturales son constructos que median entre el individuo y la sociedad y que se fundan mutuamente entre sí.

Estos postulados valorizan nuestra unidad como especie única - homo loquens, hombre que habla - al tiempo que distinguen nuestras diferencias como individuos y grupos sociales hablantes de distintas lenguas naturales. $Y$

9 MORIN, Edgar. [1999]. Los siete saberes necesarios para la educción del futuro. Bogotá: Cooperativa Editorial Magisterio, 2001. p. 112. 
sustentan que el lenguaje, mediante las lenguas naturales, es el instrumento humano constructor de la "razón y conciencia" subjetiva ${ }^{10}$, así como de los vínculos intersubjetivos que arman el tejido social.

Por su parte, la rama sociolingüística hizo otros aportes para sostener que los seres humanos son todos igualmente dignos al reconocer que:

- Todas las lenguas naturales del mundo son medios plenos de pensamiento e interacción social, independientemente de quiénes o cuántas personas las hablen, cómo o dónde.

- Las distintas variantes existentes en cualquier lengua natural surgen de que en su uso inciden factores geográficos, sociales y situacionales, sin que esto implique asignarle valoraciones desiguales en virtud de sus rasgos lingüísticos propios o de quiénes las hablen.

El primer postulado negó diferencias de valor entre lenguas "desarrolladas" $y$ "primitivas" - paralela a las diferencias entre sociedades "civilizadas" y"primitivas" que justificó la conquista y dominación colonial de muchos pueblos en la historia. El segundo, refutó los juicios peyorativos que, dentro de cada Estado, se dirigían hacia quienes usaban, por ejemplo, dialectos regionales diferentes a la variedad dominante, sociolectos poco prestigiosos, o registros situacionales descalificados y dejó en evidencia que eran meros disfraces de la discriminación hacia ciertos colectivos de hablantes para enmascarar la hegemonía política y económica de determinadas clases o sectores sociales.

Así cuestionó prejuicios vigentes sobre ciertas lenguas o variedades de lengua que, en realidad, apuntaban a sus hablantes y sus respectivas culturas. Respaldó la libertad y originalidad de las creaciones culturales y la igualdad entre ellas, que es decir, entre sus creadores.

No dudo que el estudio científico del lenguaje y las lenguas permitió conocer mejor a sus hablantes, sus facultades y creaciones, y a caracterizarlos como individuos libres, iguales y también cooperativos, en cuanto constructores de sociedades. Después, a la hora histórica de definirlos como sujetos de derechos, estos rasgos se subsumirán en la idea de dignidad, que representará lo que nos une como humanos y será la piedra basal para asentar la gran creación socio-política del siglo XX: el discurso de los derechos humanos, en constante desarrollo.

En cuanto al segundo aporte apuntado al inicio de la sección, la Lingüística y la Sociolingüística ejemplifican un discurso elaborado que reconoció nuestra semejanza esencial como familia humana - la facultad del lenguaje - en oposición y a la vez en complementación con nuestras diferencias evidentes como sociedades e individuos hablantes - las distintas lenguas 
naturales del planeta, tanto como las distintas variedades de cada lengua. Estas ciencias articularon ambos reconocimientos en un discurso dialéctico e integrador, que no negó las tensiones entre ellos, tensiones sociales e individuales, pero tampoco las presentó como irresolubles. Las exhibió, explicó y propuso políticas superadoras. De este modo impulsaron su desarrollo como ciencias y también, sostengo, el discurso de los derechos humanos.

Con un planteamiento análogo, el discurso de los derechos humanos erigió un valor abstracto como común y privativo de la especie humana - la dignidad - y lo hizo fuente de derechos iguales e irrenunciables que los Estados deben garantizar. Simultáneamente reconoció las muchas diferencias concretas que distinguen a los seres humanos en la realidad -por ejemplo de raza y etnia, sexo y género, origen nacional, lenguas y culturas, creencias religiosas, ideologías políticas, capacidades individuales y opciones de vidacomo diferencias legítimas que también merecen respeto y protección.

Tanto el discurso lingüístico como el de los derechos humanos ilustran las oposiciones entre unidad y diversidad que caracterizan las relaciones individuo/sociedad/especie del homo sapiens u homo loquens. Explicar y entender estas relaciones en las que existen rasgos opuestos en tensión, tanto en el campo de la lengua como el de los derechos humanos, es gran desafío para la vida en democracia y para la socialización de nuevas generaciones porque nuestro futuro depende de cómo se comprendan, practiquen y enseñen.

Se ha observado que es en el cruce de la triple vinculación entre individuo, sociedad y especie donde surge la conciencia o el espíritu propiamente humano y donde debe asentarse una ética con esperanza de futuro. ${ }^{11}$ Utilizando los términos más precisos del enfoque de derechos, diré que es en esa tríada donde se afirma la noción de persona o sujeto de derechos y se funda el paradigma ético y político de los derechos humanos.

\section{La mirada aplicada de la Lingüística Educativa}

Hasta aquí exploré aportes de la ciencia lingüística a la base teórica de los derechos humanos: el constructo de dignidad humana. Pero hizo mucho más. Ella también asistió y asiste para formular derechos humanos específicos reconocidos por instrumentos internacionales y regionales. Las suyas fueron y son contribuciones técnicas directas, orientadas a concretar y proteger ciertos derechos, en particular los que se relacionan con el lenguaje y la/s lengua/s. Pueden ser para definir el contenido de un derecho, para perfilar políticas públicas a fin de promoverlo en las sociedades, o para ofrecer abordajes

11 MORIN, Edgar. Op. cit. p. 112. 
y herramientas pedagógicas a la educación en derechos y corrientes afines -educación para la democracia, la paz, la ciudadanía mundial, la tolerancia y la interculturalidad, entre otras.

Acepto que ahora me desvío algo de la propuesta del Dossier, pero no puedo concluir sin hacer por lo menos algunas referencias a esta otra dimensión multidisciplinaria donde los conocimientos de la Lingüística son relevantes para avanzar hacia el "ideal común por el que todos los pueblos y naciones deben esforzarse"12, el horizonte ético que nos moviliza. A ella se lo debo por convicción y por amor. Permítanme entonces esbozar unos pocos casos ilustrativos de otras contribuciones lingüísticas que allanan caminos para efectivizar derechos humanos.

Un caso en el que ella ayuda a definir el contenido del derecho y las correspondientes obligaciones del Estado es el de los derechos lingüísticos de las minorías lingüísticas, que en la realidad enfrentan muchos obstáculos para hacerse efectivos. El uso del lenguaje y la lengua, como señalé al principio, son primordiales para los seres humanos porque son un fuerte componente de su cultura y de su identidad personal y colectiva. Además, las cuestiones lingüísticas tienen un significado emocional y social especial para las comunidades minoritarias de un país que desean mantener diferenciada su identidad cultural y de grupo, sobre todo si sufren, como ocurre a menudo, situaciones de marginalidad y discriminación.

La ciencia lingüística participa en el diálogo multidisciplinario para establecer, por una parte, el contenido de los derechos lingüísticos en general y de las minorías en particular, derechos que son a la vez individuales y colectivos, $y$, por otra parte, para fijar las responsabilidades del Estado en instrumentos internacionales de derechos humanos y otros documentos orientadores internacionales y regionales. Como ejemplo, considérense los principales problemas que afectan a las minorías lingüísticas: las amenazas a la existencia misma de los idiomas minoritarios y de las minorías lingüísticas; el reconocimiento de esos idiomas y los derechos lingüísticos de sus hablantes; el uso de esos idiomas en distintos contextos cruciales -la vida pública, la educación, los medios de comunicación, la administración pública y el sistema judicial- ; el uso de los idiomas minoritarios en los nombres personales, de lugares y en la señalización pública; la presencia de esos idiomas y la participación de su comunidad hablante en la vida económica y política del país, y la difusión de información y servicios en idiomas minoritarios. ${ }^{13}$ Sería imposible compren-

12 Declaración Universal de los Derechos Humanos (1948), Naciones Unidas, Preámbulo.

13 IZÁK-NDIAYE, Rita. Relatora Especial de Naciones Unidas sobre cuestiones de las minorías y Oficina del Alto Comisionado para los Derechos Humanos. Derechos lingüísticos de las minorías lingüísticas. Una guía práctica para su aplicación. Ginebra: Oficina de las Naciones Unidas. 2017. 
der el alcance pleno de estas cuestiones y estipular las acciones de protección que ellas demandan sin una contribución especializada de la Lingüística y sus ramas aplicadas, como la Lingüística Educativa.

Ampliando el caso anterior, nótese que cada cuestión normativa sobre la cual hay que concretar las responsabilidades estatales requiere también de asesoría experta en el diseño de políticas públicas específicas, las que pueden ser lingüísticas o educativas, aunque en esta materia ambas suelen ir de la mano.

De tal forma, otros asuntos que demandan apoyos científicos de la Lingüística Educacional, en conjunción con otras disciplinas pertinentes, son la elaboración de políticas para, por ejemplo, montar campañas de alfabetización; fomentar las competencias de lectura y escritura de la población escolar o de colectivos particulares que sufren rezagos en la materia; justificar y disponer la enseñanza bilingüe en el sistema educativo nacional o la incorporación de asignaturas sobre la enseñanza de lenguas y culturas extranjeras; razonar y promover la comunicación intercultural, y propiciar políticas editoriales y de medios de comunicación acordes con las iniciativas antes mencionadas, entre muchas otras acciones públicas posibles.

Un último caso que menciono es el de ofrecer abordajes y herramientas pedagógicas a la Educación en Derechos Humanos para destacar la importancia de la lengua y enseñar su manejo amplio y variado desde edades tempranas. Nunca destacaré lo suficiente el papel decisivo que cumple para las personas en su construcción identitaria y de un proyecto de vida digna, su convivencia social y el ejercicio de sus derechos. ¿Por qué? Porque las prácticas humanas se regulan por medio de las competencias (destrezas o habilidades) lingüísticas y comunicativas. Sin ellas, sólo nos queda recurrir a la fuerza, que es la vía opuesta a la razón. Y la razón se expresa a través del lenguaje.

En la construcción de su identidad y de su narrativa de vida las personas necesitan, por un lado, articular ideas con claridad y exactitud. Pensemos un aspecto en apariencia simple de la expresión personal: el vocabulario. Cuanto mayor riqueza y precisión léxica manejen las personas, mayores posibilidades tienen para examinarse a sí mismos y darse a entender a otros, así como de comprender a los otros. Esto significa mayores posibilidades de conocer. Paulo Freire lo expresó inmejorablemente: "conocer la palabra es conocer al mundo". ${ }^{14}$ Por otro lado, necesitan saber conectar lógica y gramaticalmente los sucesos y sus representaciones mentales en un discurso coherente, comprensible y convincente para sus interlocutores. Estos ejemplos mínimos muestran la centralidad del uso del lenguaje para elaborar y compartir nuestras experiencias vividas, nuestro mundo mental y nuestros proyectos de futuro.

14 FREIRE, Paulo. Pedagogía del oprimido, Montevideo: Tierra Nueva. 1970. 
La convivencia y el ejercicio de derechos también se benefician de buenas competencias lingüísticas y comunicativas. Primero, de las competencias básicas, las más generales: escuchar, expresarse oralmente, leer y escribir. Entre ellas son clave las competencias alfabéticas (leer y escribir) porque abren a los sujetos el acceso al medio escrito, reservorio de la cultura de la humanidad, y potencian su desarrollo cognitivo, aumentan sus oportunidades educativas, laborales, sociales y políticas, y le facilitan enfrentar desigualdades sociales de origen. Estas destrezas no son espontáneas, sino resultado del aprendizaje sistemático, escolar. Segundo, de las competencias discursivas y deliberativas, que engloban variadas destrezas particulares y focalizadas, que se apoyan a su vez en las competencias básicas: construir discursos, dialogar, justificar y demostrar, investigar, argumentar, rebatir, mediar, negociar posiciones, deliberar y un largo etc. ${ }^{15}$

Relacionado con lo anterior, hay otra cuestión sobre la cual el estudio de la lengua da una asistencia hoy más que nunca apremiante a la educación en derechos. Me refiero a develar los usos y mecanismos, a veces sutiles, del discurso discriminatorio (sea racista, sexista, paternalista, xenofóbico, homofóbico o de cualquier otro sesgo) y a enseñar a analizar los prejuicios y estereotipos que entraña a fin de de-construirlos. Así, como parte de un enfoque de derechos, el aprendizaje de la lengua está contribuyendo a una cultura de equidad y solidaridad.

En breve, de la Educación en Derechos Humanos a las disciplinas lingüísticas, jun profundo agradecimiento!

\section{5. ...en tiempos de pandemia}

Al final, rescato de estas notas la contribución de la ciencia lingüística sobre la igualdad orgánica y funcional entre las lenguas humanas por encima de sus visibles diversidades, la cual nutrió el consenso contemporáneo de la igualdad entre sus hablantes -también diversos entre sí- que sostiene el discurso de derechos humanos. Asimismo rescato los conocimientos que aporta para definir e impulsar derechos humanos particulares, igual que para desarrollar las competencias de lenguaje y comunicación de las personas como instrumentos potentes de pensamiento y acción en general y, en especial, de ejercicio y defensa de derechos.

Creo muy justo y necesario destacar estas ideas en nuestros tiempos de pandemia. Tiempos extraños y difíciles, que evidencian de manera pal-

15 RODINO, Ana María. Educación en derechos humanos para una ciudadanía democrática. San José, Costa Rica: EUNED. 2015. 
pable y desvergonzada las violaciones de derechos humanos en nuestras sociedades. Violaciones surgidas de situaciones viejas e irresueltas, como las profundas desigualdades socio-económicas entre los habitantes de nuestro continente, $\mathrm{u}$ otros lugares del mundo, y la indiferencia hacia los colectivos en condiciones de vulnerabilidad.

Hoy los más pobres y desprotegidos no pueden cumplir con el distanciamiento social y las normas de higiene recomendadas cuando viven precariamente, en hacinamiento y sin servicios básicos de agua potable, cloacas y electricidad; o poner al alcance de sus niños los recursos tecnológicos y la conectividad a fin de sostener, aunque sea con las uñas, el derecho a la educación frente a escuelas cerradas; $u$ obtener prontos servicios de salud para ser testeados y atendidos por el sistema sanitario público si resultan infectados; o acceder a fuentes de sustento si pierden su trabajo o su ingreso depende de actividades informales que realizan en el día a día en las calles... La pandemia pone en foco estas situaciones, las exhibe brutalmente sin disimulo $Y$ demuestra -por si alguna duda quedara- que la inequidad mata. Arrebata la alegría de vivir, la educación, la salud, el sustento y hasta la vida de muchas, demasiadas personas, por lo habitual las más necesitadas de cada sociedad

Por esto hoy revalorizo los aportes de mi entrañable Lingüística a la idea de la igualdad entre humanos como uno de los puntales del constructo de dignidad humana. Más allá de que la dignidad se entienda como un consenso ético e histórico -posición que suscribo-, o que se la conciba como un rasgo natural y preexistente de la especie humana, creo que debemos seguir haciendo de ella una bandera de lucha y enarbolándola muy alta porque en estos tiempos de pandemia necesitamos, más que nunca, defenderla con todas las herramientas de la razón que tengamos a nuestro alcance. 
